\title{
Modelling a Job-Shop Plant Using Queuing Networks
}

\section{Techniques}

\author{
Artur Barreiros \\ Mechanical Engineering Department, Instituto Superior Técnico, Av. Rovisco Pais, 1049-001Lisbon, Portugal
}

Received: September 12, 2013 / Accepted: September 15, 2013 / Published: September 25, 2013.

\begin{abstract}
This article illustrates the use of open queuing networks for the planning of discrete production systems of the job-shop type. For this purpose, it is assumed that the system can be modelled by a set of workstations. The mathematical and numerical procedures, related to the definition of the model that is used to characterize the system operations, are analyzed first. For this purpose, an ideal situation of a system with only two workstations and four products is used. Next, the application of the model to a real case study, corresponding to a metalworking company, is illustrated. In this case, the parameters that can be used to characterize the efficiency of the system are also introduced. The performance diagnostics involves the analysis of the work-in-process and the production lead-times. The optimization of the system involves the probabilistic characterization of the demand and the characterization of the probability of transference of products among workstations. Among other alternatives, a waiting-cost function, which is evaluated using the queuing parameters, can be used to compare alternative solutions.
\end{abstract}

Key words: Queuing Networks, job-shop plant, optimization.

\section{Introduction}

This article aims to illustrate the application of open queuing networks techniques to characterize, from the operational point of view, production companies of the discrete job-shop type. The importance of conducting this type of study is justified due to the competitive environment in which the majority of these companies operate. Through this type of study it is possible to identify changes in the organizational structure that will enable the company to gain greater flexibility to deal with market uncertainties. Additionally, in this way the company will have greater capacity for innovation and other competitive advantages over the competition.

To carry out this study, a real situation corresponding to a metalworking company, operating in the job-shop system, is used. Data corresponding to one year of production, involving 9 product classes and

Corresponding author: Artur Barreiros, Ph.D., research field: industrial engineering and operations research. E-mail: barreiros@ist.utl.pt.
10 workstations, is used. The job-shop system is a form of organization of the production system that allows the processing of various classes of products, however, having capacity limitations inherent to the control and handling of the products and the preparation time of the machines (setup-time). The machines of the system can perform operations on different classes of products which involve higher setup-times, higher lead-times and higher levels of work in progress.

There are several alternatives for the evaluation of systems with these characteristics (e.g. Refs. [1, 2]). These alternatives include the parametric decomposition method, the diffusion approximation, the operational analysis, and the analysis of the average value. The discrete simulation can also be used, especially in the absence of analytical results, or approximations compatible, for example, with the constraints of the problem. In this study, approximations based on queuing networks, with models of type M/M/1 for each workstation, are used.

This choice is motivated by the randomness in 
demand and production process. The consideration of an open queuing system results from the fact that the number of products in circulation in the network is a random variable, which is influenced by the arrival or departure of products. In this case, each machine or workstation is modelled as a queuing system for which it matters to know the arrival rate of products (jobs) and the average processing capacity. Nevertheless, to achieve these tasks, it is necessary to know the average flow among workstations.

The study of queuing problems has its origin in problems related to the telephone industry [3]. Subsequently, its application was extended to other areas, namely to transport and production, among many others [4]. Comprehensive descriptions of the use of queuing networks for the representation of production systems can be found in Refs. [2, 5], but the processes to define the network parameters are not a priority in these studies.

These models can be used for performance evaluation or to optimize the configuration of the production system (e.g. [6]). The focus of this study is the performance evaluation, which provides detailed information about the operation of the system for a given configuration, and allows the evaluation of the impact of changes in external conditions. Results of this study were presented in Ref. [7]. In this article it is intended to present a detailed definition and description of the mathematical model, along with the definition of new parameters to characterize the operations of the system.

This paper is organized as follows. The next section presents the mathematical formulation of typical open queuing network problems. For this purpose, a simple situation is used to illustrate the evaluation of the arrival rate of products to each workstation and the average flows among workstations. The third section is devoted to the presentation of the case study and evaluation of the model parameters, introduced in the previous section, for the characterization of the typical operating conditions. Additional parameters, which can be used in the optimization procedure, are also introduced. The following section is related to the presentation and discussion of results. The last section summarizes the main conclusions of this work.

\section{Mathematical Modelling}

In this section a simple situation, corresponding to a production system with two workstations and four different products, is analyzed. The system representation, illustrating the production trajectory of each product, is shown in the following figure. The products $\mathrm{P} 1$ and $\mathrm{P} 2$ begin the manufacturing process at the workstation S1, while the products P3 and P4 start the process at the workstation S2. The product P1 only needs to go through workstation S1. The product P2, after S1, is also processed in the workstation S2. The product P4 only needs to pass through the workstation S2. The product P3, after S2, goes to the workstation S1. The rates of arriving of products to the system, related to the demand, are: $\lambda_{P}=\{1,7,6,11\}$ unit/h.

The aim is to determine the rates of arriving of products at each workstation. The rates of arriving of products coming from the exterior can be evaluated directly: for the workstation S1, it is obtained $r_{1}=\lambda_{P 1}+\lambda_{P 2}=8 u$ unit/h, and for the workstation S2 it is obtained $r_{2}=\lambda_{P 3}+\lambda_{P 4}=17$ unit/h. In this simplified situation, the rate of arriving of products to the workstation $\mathrm{S} 1$ is $\lambda_{1}=r_{1}+\lambda_{P 3}=14 \mathrm{unit} / \mathrm{h}$, and the

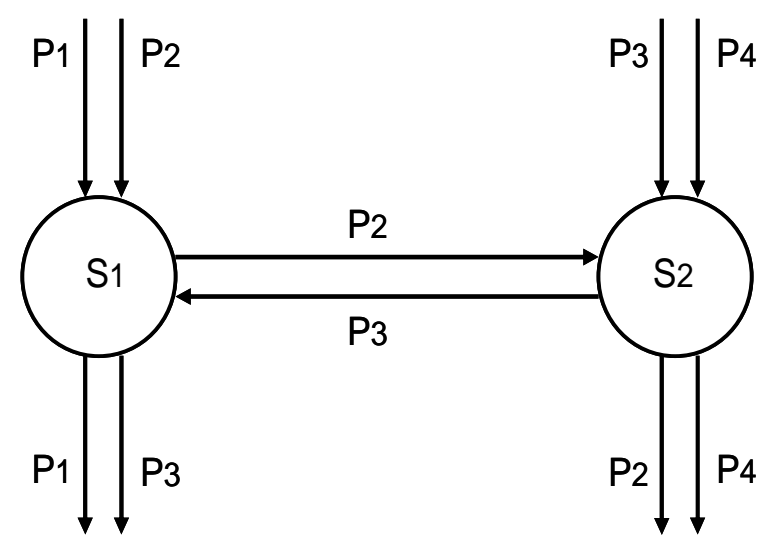

Fig. 1 Production system with 2 workstations and 4 products. 
rate of arriving of products to the workstation S2 is $\lambda_{2}=r_{2}+\lambda_{P 2}=24$ unit/h. From the analysis of this example, it is easy to conclude that, in general, the solution of this problem can be obtained from the Eq. (1):

$$
\left[I-P^{T}\right] \lambda=r
$$

where $I$ is the identity matrix and $P$ is the transference matrix, whose elements represent the fraction of products that pass from one workstation to another. In our example, the terms of this matrix can be evaluated directly, applying the control volume balance technique to each workstation, which gives: $P_{12}=\lambda_{P 2} / \lambda_{1}=0.5, \quad P_{21}=\lambda_{P 3} / \lambda_{2}=0.25$ and the system of equations becomes:

$$
\left[\begin{array}{cc}
1 & -0.25 \\
-0.5 & 1
\end{array}\right]\left[\begin{array}{l}
\lambda_{1} \\
\lambda_{2}
\end{array}\right]=\left[\begin{array}{c}
8 \\
17
\end{array}\right]
$$

The solution of the system, as was already presented, is: $\left(\lambda_{1}, \lambda_{2}\right)=(14,24)$.

In more complex systems, with multiple workstations and multiple products, the determination of the transference matrix, $P$, and of the vector of rates of arriving of products from the exterior, $r$, has to be performed automatically. For this purpose, it is proposed in this study to define first a matrix of trajectories of products, $T(k, l)$, where $k$ is the index for product identification, $k=1, . ., n p$, and $l$ is the index identifying the sections traversed, $l=1, . ., n s p(k)$, being $n p$ the number of products considered and $n s p(k)$ the number of sections traversed by the product $k$. Next, it is defined a vector that counts the inputs per unit of time at each workstation, $N E(i)$, and an array that counts the entries in the workstation $j$ coming from the workstation $i, N S(i, j)$, where $i=1, . ., n w$ and $j=1, . ., n w$, being $n w$ the number of workstations of the system.

The terms of the vector of arrivals coming from the exterior, are determined from the following set of expressions, defined using standard programming language conventions:

$$
\left\{\begin{array}{l}
i=T(k, 1) \\
r(i)=r(i)+\lambda_{P k}
\end{array}\right\}, k=1, . ., n p
$$

The evaluations of the vector of inputs per unit of time, $N E$, and the matrix of entries from other workstations, NS, are obtained from the set of Eq. (4), while the terms of the transference matrix, $P$, are evaluated from the terms of $N E$ and $N S$, using the Eq. (5).

$$
\begin{gathered}
\left\{\begin{array}{l}
i=T(k, l) \\
j=T(k, l+1) \\
N E(i)=N E(i)+\lambda_{P k} \\
N S(i, j)=N S(i, j)+\lambda_{P k}
\end{array}\right\}, \\
k=1, . ., n p ; l=1, . ., n s p(k) \\
P(i, j)=N S(i, j) / N E(i)
\end{gathered}
$$

\section{The Case Study}

The case study used in this work is a company in the business of metalworking, located in the Lisbon area, which has nearly thirty years of activity and that works in a two-story building with about $720 \mathrm{~m}^{2}$ of total floor area. The relevant data for applying the model, shown in the previous section, is related to its activity in a recent year, and is presented in Table 1.

The data in Table 1 is related to nine major products that are produced involving 10 workstations. The description of these workstations, which were numbered arbitrarily, is as follows: in workstation S1 are carried out operations with a guillotine, in S2 a press brake is used, S3 is a drilling machine, S4 is a milling cutter, S5 and S6 are turning machines, S7 is dedicated to sawing, S8 is for welding, S9 is dedicated to grinding and S10 to painting.

To clarify the information provided by this data, for example for product P1, it is stated that 125 units, distributed by 8 orders, were produced in that year. Additionally, to produce a unit of P1 workstation S6 is used firstly and then the product is finalised in S4. 
To use the queuing model it is also needed to evaluate the mean service times and the setup times of the products on each workstation. This information was obtained by performing measurements on site and it is presented in Tables 2-3.

It should be noted that the production time for each product in each section, presented in Table 2, corresponds to the average of all the product passes by the section. Accordingly, the number of products that passes through each section, per unit of time, also accounts for the passages following the first. For example, the number of products of the type P3 processed in sections S2, S3, S8 and S9, is 87, while in sections S6 and S7 is considered to be 174, corresponding to two passages through these sections.

With regard to the setup time of each product in each section, presented in Table 3, it corresponds to the total time of all setups required per order in the section.

With this information, it can be determined the average processing time of products in each workstation, $T p$, and the mean rate of service of each workstation, $\mu$, using the following expressions:

Table 1 List of products, number of orders, annual demand, and range of manufacturing.

\begin{tabular}{llll}
\hline Product & Orders & Units & Work-stations \\
\hline P1 & 8 & 125 & S6 S4 \\
P2 & 8 & 84 & S6 S7 S3 S8 \\
P3 & 8 & 87 & S7 S6 S7 S2 S3 S6 S8 S9 \\
P4 & 5 & 83 & S5 S7 S8 \\
P5 & 7 & 76 & S1 S5 S3 S3 S5 S5 \\
P6 & 7 & 61 & S5 \\
P7 & 7 & 60 & S5 \\
P8 & 3 & 60 & S7 S6 S8 S1 S6 S10 \\
P9 & 7 & 110 & S7 S4 S3 S10 S6 S6 S7 S6 S8 S6 S7 S6 S10
\end{tabular}

Table 2 Production time of the products on the workstations (min).

\begin{tabular}{lllllllrrrr}
\hline & S1 & S2 & S3 & S4 & S5 & S6 & S7 & S8 & S9 & S10 \\
\hline P1 & - & - & - & 9.5 & - & 20.0 & - & - & - & - \\
P2 & - & - & 25.0 & - & - & 47.5 & 16.0 & 121.5 & - & - \\
P3 & - & 1.0 & 6.0 & - & - & 10.0 & 5.0 & 6.0 & 7.0 & - \\
P4 & - & - & - & - & 46.0 & - & 16.0 & 40.5 & - & - \\
P5 & 5.0 & - & 20.0 & - & 8.3 & - & - & - & - & - \\
P6 & - & - & - & - & 13.0 & - & - & - & - & - \\
P7 & - & - & - & - & 23.0 & - & - & - & - & - \\
P8 & 5.0 & - & - & - & - & 12.5 & 10.0 & 8.0 & - & 10.0 \\
P9 & - & - & 12.0 & 12.0 & - & 21.0 & 7.7 & 15.0 & - & 10.0 \\
\hline
\end{tabular}

Table 3 Setup time in the workstations for each type of products (min).

\begin{tabular}{lcccccccccc}
\hline & S1 & S2 & S3 & S4 & S5 & S6 & S7 & S8 & S9 & S10 \\
\hline P1 & - & - & - & 15.0 & - & 13.5 & - & - & - & - \\
P2 & - & - & 0.0 & - & - & 62.5 & 5.0 & 0.0 & - & - \\
P3 & - & 10.0 & 0.0 & - & - & 15.0 & 15.0 & 0.0 & 5.0 & - \\
P4 & - & - & - & - & 15.0 & - & 10.0 & 3.0 & - & - \\
P5 & 10.0 & - & 20.0 & - & 24.0 & - & - & - & - & - \\
P6 & - & - & - & - & 7.0 & - & - & - & - & - \\
P7 & - & - & - & - & 7.0 & - & - & - & - & - \\
P8 & 10.0 & - & - & - & - & 10.0 & 10.0 & 0.0 & - & 20.0 \\
P9 & - & - & 5.0 & 15.0 & - & 40.0 & 25.0 & 5.0 & - & 40.0 \\
\hline
\end{tabular}




$$
\begin{array}{r}
T p_{j}=\frac{\sum_{i=1}^{n p}\left(T m_{i j} n_{i j}+T s_{i j} n s t_{i j}\right)}{\sum_{i=1}^{n p} n_{i j}} \\
\mu_{j}=\frac{1}{T p_{j}}, \quad j=1, \ldots, n w
\end{array}
$$

where $\mathrm{Tm}_{i j}$ is the mean processing time of products of type $i$ in machine $j, n_{i j}$ is the total number of products of type $i$ processed in machine $j . T s_{i j}$ is the total setup time of product $i$ in machine $j$, and $n s t_{i j}$ is the number of setups in machine $j$ for products of type $i$.

In real conditions, to characterize the operation of the system, it is also needed to take into account the time of transport of products between workstations. For this purpose measurements were made on site to assess the average time between two workstations, considering all combinations. The results of these measurements are presented in Table 4.

Accordingly, to characterize the performance of the system the mean residence time of the products in each workstation, $W$, is used, and as a complement, it is also used the average time spent in the system under the influence of each workstation, WT, whose evaluation is obtained from the following expression:

$$
W T_{j}=W_{j}+T d_{j}, \quad j=1, \ldots, n w
$$

where:

$$
T d_{j}=\sum_{k=1}^{n w+1} p_{j k} T t_{j k}
$$

That is, the mean residence time in the system in the influence of each station is defined adding to the mean residence time of the products in the workstation, the mean transference time, $T d$, form each station to the other stations and to the exit, which is evaluated using the transference matrix and the matrix of the transportation times.

\section{Results and Discussion}

This section presents the results obtained with the model described in the previous sections, for the characterization of the operating conditions of the metalworking company. The operating resources and the typical demand of products are described in the previous section.

To present the properties for assessing the operating conditions of each workstation, it is necessary to evaluate first the transference matrix. Considering the data presented in Table 1, and the Eqs. (4) and (5) the results presented in Table 5 are obtained.

To evaluate the queuing properties of each workstation, it is necessary to evaluate also the vector of rates of arriving of products from the exterior to each workstation, using the set of Eq. (3), and then evaluate the total rate of products arriving to each workstation, solving the system of equations defined in Eq. (1). The results obtained in this way are shown in Table 6.

The results in Table 6 show that the products enter the system from the stations S1, S5, S6 and S7 and that the workstations with greater solicitation (i.e. greater than the average) are the following: S3, S5, S6, S7 and S8. The conversion of the value of the arriving rates of products from the annual time base to minutes was performed assuming that each month has 22 working days and each day has 8 hours of work.

The parameters of the queuing model, as the occupation rate, $\rho$, the mean number of elements in the workstation, $L$, and the mean residence time in the workstation, $W$, are also a function of the mean service rate. The average service time and the average rate of service, for each workstation, are shown in the following Table 7 and are evaluated using the data of Tables 1-3 and the Eqs. (6) and (7).

In the Table 7 the values of $T m$ represent the average processing time of a unit of product, Ts represents the component of the time of production associated to the setup of the machines, and $T p$ represents the mean production time. From these results, it is concluded that stations S3, S5, S6 and S8 are those with higher mean production times. 
Table 4 Transportation time between workstations ( $\mathrm{min}$ ).

\begin{tabular}{lccccccccccc}
\hline & S1 & S2 & S3 & S4 & S5 & S6 & S7 & S8 & S9 & S10 & Exit \\
\hline S1 & - & 5 & 5 & 5 & 8 & 8 & 25 & 25 & 25 & 25 & - \\
S2 & 5 & - & 5 & 5 & 7 & 7 & 25 & 25 & 25 & 25 & - \\
S3 & 5 & 5 & - & 5 & 5 & 6 & 25 & 25 & 25 & 25 & - \\
S4 & 5 & 5 & 5 & - & 8 & 6 & 25 & 25 & 25 & 25 & 5 \\
S5 & 8 & 7 & 5 & 8 & - & 5 & 25 & 25 & 25 & 25 & 5 \\
S6 & 8 & 7 & 6 & 6 & 5 & - & 25 & 25 & 25 & 25 & - \\
S7 & 25 & 25 & 25 & 25 & 25 & 25 & - & 5 & 5 & 8 & - \\
S8 & 25 & 25 & 25 & 25 & 25 & 25 & 5 & - & 5 & 5 & 5 \\
S9 & 25 & 25 & 25 & 25 & 25 & 25 & 5 & 5 & - & 5 & 5 \\
S10 & 25 & 25 & 25 & 25 & 25 & 25 & 8 & 5 & 5 & - & 5 \\
\hline
\end{tabular}

Table 5 Transference matrix (fraction transferred from $S i$ to $S j$ ).

\begin{tabular}{llllllllllll}
\hline & S1 & S2 & S3 & S4 & S5 & S6 & S7 & S8 & S9 & S10 & EXIT \\
\hline S1 & - & - & - & - & 0.559 & 0.441 & - & - & - & - & - \\
S2 & - & - & 1.000 & - & - & - & - & - & - & - & - \\
S3 & - & - & 0.176 & - & 0.176 & 0.200 & - & 0.194 & - & 0.254 & - \\
S4 & - & - & 0.468 & - & - & - & - & - & - & - & 0.532 \\
S5 & - & - & 0.176 & - & 0.176 & - & 0.192 & - & - & - & 0.456 \\
S6 & - & - & - & 0.119 & - & 0.105 & 0.371 & 0.244 & - & 0.161 & - \\
S7 & - & 0.119 & 0.115 & 0.150 & - & 0.502 & - & 0.114 & - & - & - \\
S8 & 0.142 & - & - & - & - & 0.259 & - & - & 0.205 & - & 0.394 \\
S9 & - & - & - & - & - & & - & - & - & - & 1.000 \\
S10 & - & - & - & - & - & 0.393 & - & - & - & - & 0.607 \\
\hline
\end{tabular}

Table 6 Rates of arriving of products to the workstations: from the exterior and global.

\begin{tabular}{lcccccccccrr}
\hline & S1 & S2 & S3 & S4 & S5 & S6 & S7 & S8 & S9 & S10 \\
\hline$r\left(\right.$ year $\left.^{-1}\right)$ & 76.0 & 0.0 & 0.0 & 0.0 & 204.0 & 209.0 & 257.0 & 0.0 & 0.0 & 0.0 \\
$\lambda\left(\right.$ year $\left.^{-1}\right)$ & 136.2 & 86.9 & 433.0 & 234.6 & 432.4 & 1051.5 & 730.1 & 423.8 & 86.9 & 279.3 \\
$\lambda\left(\right.$ min $\left.^{-1}\right)$ & 0.0011 & 0.0007 & 0.0034 & 0.0019 & 0.0034 & 0.0083 & 0.0058 & 0.0033 & 0.0007 & 0.0022 \\
\hline
\end{tabular}

Table 7 Average service time and average rate of service.

\begin{tabular}{lllllrrrrrr}
\hline & S1 & S2 & \multicolumn{1}{c}{ S3 } & \multicolumn{1}{c}{ S4 } & S5 & \multicolumn{1}{c}{ S6 } & S7 & S8 & S9 & S10 \\
\hline Tm (min) & 5.00 & 1.00 & 16.12 & 10.67 & 18.27 & 20.21 & 9.13 & 38.25 & 7.00 & 10.00 \\
$T s$ (min) & 0.74 & 0.92 & 0.40 & 0.96 & 0.79 & 0.99 & 0.57 & 0.12 & 0.46 & 1.21 \\
$T p(\min )$ & 5.74 & 1.92 & 16.53 & 11.63 & 19.06 & 21.19 & 9.69 & 38.37 & 7.46 & 11.21 \\
$\mu\left(\mathrm{min}^{-1}\right)$ & 0.1744 & 0.5210 & 0.0605 & 0.0860 & 0.0525 & 0.0472 & 0.1031 & 0.0261 & 0.1341 & 0.0892 \\
\hline
\end{tabular}

The functions that define the parameters $\rho, L$ and $W$ are available, for example in Ref. [8]. Using these expressions with the results presented in Table 7-8, and the Eq. (8) and (9), the results shown in Table 8 are obtained. Table 8 also shows results for an additional parameter, $R$, which is the ratio between the mean residence time in the system, in the influence of a workstation, and the time required to process a unit of product, in the workstation considered. The expression for the evaluation of this parameter is as follows:

$$
R=\mu W T
$$

The information provided by this parameter identifies the most requested workstations in terms of length of service and ease of product movement. In this case, it becomes evident that to change the actual situation of a workstation, the decision maker can act in the factors that influence one of those times, or both. 
Table 8 Performance parameters of the job-shop.

\begin{tabular}{lllllllllll}
\hline & S1 & S2 & S3 & S4 & S5 & S6 & S7 & S8 & S9 & S10 \\
\hline$\lambda\left(\mathrm{min}^{-1}\right)$ & 0.0011 & 0.0007 & 0.0034 & 0.0019 & 0.0034 & 0.0083 & 0.0058 & 0.0033 & 0.0007 & 0.0022 \\
$\mu\left(\mathrm{min}^{-1}\right)$ & 0.1744 & 0.5210 & 0.0605 & 0.0860 & 0.0525 & 0.0472 & 0.1031 & 0.0261 & 0.1341 & 0.0892 \\
$\rho(\%)$ & 0.62 & 0.13 & 5.65 & 2.15 & 6.50 & 17.59 & 5.59 & 12.83 & 0.51 & 2.47 \\
$L$ (units) & 0.006 & 0.001 & 0.060 & 0.022 & 0.070 & 0.213 & 0.059 & 0.147 & 0.005 & 0.025 \\
$W$ (min) & 5.77 & 1.92 & 17.52 & 11.88 & 20.38 & 25.72 & 10.27 & 44.02 & 7.50 & 11.50 \\
$T d$ (min) & 8.00 & 5.00 & 13.28 & 5.00 & 5.68 & 20.11 & 22.72 & 13.02 & 5.00 & 9.83 \\
$W T$ (min) & 13.77 & 6.92 & 30.80 & 16.88 & 26.06 & 45.83 & 32.99 & 57.04 & 12.50 & 21.32 \\
$R$ & 2.40 & 3.61 & 1.86 & 1.45 & 1.37 & 2.16 & 3.40 & 1.49 & 1.68 & 1.90 \\
\hline
\end{tabular}

The results presented in Table 8 show that the residence time, $W$, of the workstations is greater than the average, at stations S3, S5, S6, and S8. However, when it is taken into account the transportation time of the products stands out, additionally, the workstation S7, and workstation S5 becomes below the average. Workstations S1, S2, S6 and S7 are those with higher values of the parameter $R$, but in the cases of $\mathrm{S} 1$ and $\mathrm{S} 2$, this parameter is high because the processing time is low, which means that these workstations are not targeted for improvement.

\section{Conclusions}

In this article, the use of open queuing networks techniques to characterize the operations of discrete production systems of the job-shop type is illustrated. The methodology is defined starting with a simple theoretical example, considering a system with only two-workstations, and then a real test case is introduced, which allows to establish additional modelling requirements related with practical situations.

The case study used in this work is related to a company in the business of metalworking, and the data used for modelling purposes corresponds to one year of activity. The use of this case study allows the identification of a decisive variable for the modelling process: it is verified that the behaviour of the system can be described from the matrix of transference of components between workstations, which can be evaluated from the historical data.
The results presented show that the modelling process using open queuing networks, permits to identify the most loaded workstations and the workstations with a poor location. Additionally, assuming that the structure of the demand is stable, it can be concluded that this methodology allows the estimation of the impact of changes in demand, and the planning of the necessary steps to optimize the system response.

\section{References}

[1] G.R. Bitran, D. Tirupati, Multiproduct queueing networks with deterministic routing: Decomposition approach and the notion of interference, Management Science 34 (1) (1988) 75-100.

[2] R. Suri, J.L. Sanders, M. Kamath, Performance evaluation of production networks, Handbooks in OR/MS, 1993.

[3] A.K. Erlang, Solution of some problems in the theory of probabilities of some significance in automatic telephone exchanges, Post Office Electrical Engineer's Journal 10 (1917) 189-197.

[4] R.L. Disney, D. Konig, Queueing networks: A survey of their random processes, SIAM Review 27 (3) (1985) 335-403.

[5] L.F. Hsu; C.S. Tapiero, C. LIN, Network of queues modeling in flexible manufacturing systems: A survey, RAIRO 27 (2) (1993) 201-248.

[6] G.R. Bitran, S. Dasu, A review of open queueing network models of manufacturing systems, Queueing Systems 12 (1992) 95-134.

[7] A. Barreiros, S. Dulce, Optimizing a job-shop plant using queuing networks techniques, in: 24th European Conference on Operational Research, University of Lisbon-Faculty of Sciences, Lisbon, Portugal, 2010.

[8] F.S. Hillier, G.J. Lieberman, Introduction to Operations Research, McGraw-Hill, USA, 2001. 